\title{
Information and Two-Sided Platform Profits
}

\section{Citation}

Hagiu, Andrei, and Hanna Halaburda. "Information and Two-Sided Platform Profits."

International Journal of Industrial Organization 34 (May 2014): 25-35.

\section{Published Version}

http://www.sciencedirect.com/science/article/pii/S0167718714000411

\section{Permanent link}

http://nrs.harvard.edu/urn-3:HUL.InstRepos:14358173

\section{Terms of Use}

This article was downloaded from Harvard University's DASH repository, and is made available under the terms and conditions applicable to Open Access Policy Articles, as set forth at http:// nrs.harvard.edu/urn-3:HUL.InstRepos:dash.current.terms-of-use\#OAP

\section{Share Your Story}

The Harvard community has made this article openly available.

Please share how this access benefits you. Submit a story.

\section{Accessibility}




H A R V A R D

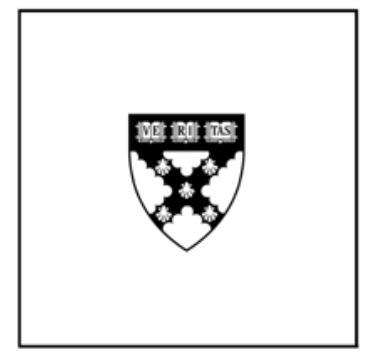

\title{
Information and Two-Sided Platform Profits
}

\author{
Andrei Hagiu \\ Hanna Hałaburda
}

\section{Working Paper}

12-045

April 5, 2014 


\title{
Information and Two-Sided Platform Profits*
}

\author{
Andrei Hagiu ${ }^{\dagger} \quad$ Hanna Hałaburda ${ }^{\ddagger}$
}

April 5, 2014

\begin{abstract}
We study the effect of different levels of information on two-sided platform profitsunder monopoly and competition. One side (developers) is always informed about all prices and therefore forms responsive expectations. In contrast, we allow the other side (users) to be uninformed about prices charged to developers and to hold passive expectations. We show that platforms with more market power (monopoly) prefer facing more informed users. In contrast, platforms with less market power (i.e., facing more intense competition) have the opposite preference: they derive higher profits when users are less informed. The main reason is that price information leads user expectations to be more responsive and therefore amplifies the effect of price reductions. Platforms with more market power benefit because higher responsiveness leads to demand increases, which they are able to capture fully. Competing platforms are affected negatively because more information intensifies price competition.

Keywords: two-sided platforms, information, responsive expectations, passive expectations, wary expectations
\end{abstract}

${ }^{*}$ We are grateful to Andres Hervas-Drane, Bruno Jullien, Robin Lee, Gaston Llanes, Francisco RuizAliseda, Richard Schmalensee, Julian Wright and Liyan Yang for very helpful comments on earlier drafts of this paper. All remaining errors are our own.

${ }^{\dagger}$ Harvard University (HBS Strategy Unit), ahagiu@hbs.edu.

${ }^{\ddagger}$ Bank of Canada, hhalaburda@gmail.com. Views presented in this paper are those of authors, and do not represent Bank of Canada's position. 


\section{Introduction}

In markets with two-sided network effects, the value that an agent derives from joining a platform is determined by the number of agents on the other side (cross-group network effects). Examples include payment systems like PayPal or Visa, videogame systems like PlayStation 3 and Xbox 360, smartphone platforms like Apple's iPhone or Google's Android, etc. Because of this cross-group dependency, the number of agents that join each side ultimately depends on the prices charged to both sides. In most real-world settings, however, some agents may not take all prices into account when forming expectations. This may be for a number of reasons. One side (typically consumers) may simply not be informed of the price charged to the other side. For instance, few videogame console users are aware of the royalties that console manufacturers charge to third-party game developers. Few iPhone users are aware of the fees charged by Apple to third-party app developers. And even when all prices are known to everyone, consumers may not have sufficient information about aggregate demand on each side to compute each demand's responsiveness to price changes. Instead, consumers typically rely on external information (e.g., press announcements, market reports, word of mouth) to form expectations about the total number of developers that join a given platform. Expectations formed in this way usually do not respond to changes in platform prices.

The majority of the existing literature on two-sided platform pricing typically assumes that agents on all sides have full information about all prices and are able to perfectly compute their impact on platform adoption. In other words, expectations adjust perfectly in response to changes in platform prices. In this paper we demonstrate that different levels of information - which imply different mechanisms through which agents form rational expectations - lead to economically meaningful differences in terms of market outcomes, firm profits in particular.

For convenience, we label the two sides as "users" and "developers" (e.g., smartphone operating systems, videogame consoles). Throughout the paper we assume that the developer side has full information about all prices and therefore always forms responsive expectations about user participation. In contrast, we allow the user side to be uninformed about developer prices and we assume - as is realistic - that uninformed users form passive expectations about developer participation. In other words, when users are uninformed about developer prices, they use external information to fix their expectation of platform adoption by devel- 
opers and do not adjust it in response to any price changes. Nevertheless, in equilibrium passive expectations are fulfilled, i.e. are rational.

Our main results and insights are derived by comparing the scenarios with fully informed users and fully uninformed users, for a monopoly platform and for competing platforms. We show that a monopoly platform's profits are higher when users are informed relative to the case when users are uninformed. In contrast, the ranking of platform profits in a symmetric duopoly equilibrium with fixed user market size is exactly the opposite. Competing platforms in such a setting prefer to face uninformed rather than informed users.

We also provide several extensions of our baseline model. In one extension, we analyze the case when only a fraction of users are informed, while the rest are uninformed. As expected, monopoly platform profits are increasing in the fraction of informed users, while competing platform profits are decreasing in that fraction. Furthermore, a generalized model of competition confirms the insights derived from our baseline model: equilibrium profits are larger with uninformed users than with informed users if and only if competition for users is sufficiently intense.

In another extension, we allow uninformed users to hold wary expectations instead of passive expectations. Wary expectations are formed when users expect the platform to charge a developer price that is optimal conditional on the observed user price. It is worth emphasizing that wary expectations are also rational, i.e., fulfilled in equilibrium. When uninformed users hold wary expectations, we obtain equilibrium platform profits that are in-between those with informed users and uninformed users forming passive expectations, both under monopoly and under platform competition with fixed market size. In other words, competing platforms continue to have the opposite preference regarding user information relative to a platform monopolist.

The broader implication of our analysis is that, in two-sided markets with cross-group network effects, the more market power platforms have, the more they prefer facing agents that are informed about prices on all sides. In contrast, platforms with less market power (due to more intense competition) prefer facing uninformed agents. Indeed, due to network effects, information about prices and price changes leads agents on one side to adjust their expectations about participation on the other side. Thus, the presence of informed agents tends to amplify the effect of price reductions. Platforms with more market power benefit because this leads to demand increases, which they are able to capture fully. Platforms which 
face more intense competition suffer because the presence of informed agents intensifies price competition.

\section{Related literature}

The existing literature on platform pricing in the presence of network effects (one-sided and two-sided) contains two different approaches to modeling rational expectations: ${ }^{1}$ responsive and passive. Models with responsive expectations implicitly assume agents on all sides are informed of all prices and capable of perfectly computing the effect of price changes on overall demands for a given platform. In other words, expectations adjust perfectly to match realized demands for all prices. Conversely, models with passive expectations assume that agents hold their expectation of total demand (on the same side for one-sided networks or on the other side for two-sided networks) fixed, irrespective of platforms' price choices. Rationality is then obtained by imposing that fixed expectations are correct at the equilibrium prices.

We focus our analysis on two-sided platforms. When agents on at least one side are uninformed of the prices on the other side, passive expectations can be part of subgame perfect equilibria. In other words, passive expectations are not "incorrect" when firm prices deviate from the equilibrium path, simply because uninformed agents do not observe price deviations on the other side. ${ }^{2}$ By contrast, in models with one-sided network effects, in order to make passive expectations compatible with subgame perfection, it is necessary to additionally assume that expectations are formed and observed by firms prior to their pricing decisions (cf. Matutes and Vives 1996). ${ }^{3}$ In other words, customers must fix their expectations before firms set their prices (even though customers make purchase decisions after observing firm prices) and firms must observe those expectations.

This observation provides further justification for our focus on two-sided platforms, and on the distinction between informed and uninformed users. In particular, in our model informed users always form responsive expectations. For uninformed users, we assume throughout most of the paper that they form passive expectations, i.e., they keep their expectations

\footnotetext{
${ }^{1}$ In models of platform adoption with network effects where platforms do not make pricing decisions (e.g., Church and Gandal (1992), Farrell and Saloner (1985) and (1986)), the distinction between various types of rational expectations does not make a difference.

${ }^{2}$ We are grateful to an anonymous referee for pointing this out.

${ }^{3}$ Passive expectations may be part of a Nash equilibrium in models with one-sided network effects without this additional assumption, but then they would not satisfy subgame perfection.
} 
fixed regardless of the price they are charged (the only price they observe). In section 6.1 we also analyze the case in which uninformed users hold wary expectations, i.e. they adjust expectations based on the price they observe.

Most models of platform pricing with network effects rely on responsive expectations. This approach originated with Katz and Shapiro (1986), who study competition between incompatible technologies with direct (one-sided) network effects. A majority of the recent literature on two-sided markets (e.g., Armstrong (2006), Armstrong and Wright (2007), Caillaud and Jullien (2003), Choi (2010), Hagiu (2009), Halaburda and Yehezkel (2013), Rochet and Tirole (2006), Weyl (2010)) also assumes responsive expectations. The typical analysis of a monopoly two-sided platform runs as follows. For the two sides $i=1,2$, the demand functions are given by $n_{i}=D_{i}\left(n_{j}, p_{i}\right)$, where $n_{j}$ denotes the realized demand on side $j \neq i$ and $p_{i}$ denotes the price charged by the platform to side $i ; D_{i}(.,$.$) is increasing in its first$ argument and decreasing in its second argument. Then all authors adopt one of two methods. They either solve directly for $\left(n_{1}, n_{2}\right)$ as a function of $\left(p_{1}, p_{2}\right)$ only, and then maximize the resulting profit expression over $\left(p_{1}, p_{2}\right)$. Or they invert $n_{i}=D_{i}\left(n_{j}, p_{i}\right)$ to express $p_{i}$ as a function of $\left(n_{i}, n_{j}\right)$, then replace in the formula for profit, and maximize over $\left(n_{1}, n_{2}\right)$. The problem (which arises with both methods) is that there may be multiple equilibrium solutions $\left(n_{1}, n_{2}\right)$ for a given $\left(p_{1}, p_{2}\right)$. This issue is usually side-stepped by assuming platforms have the ability to coordinate users on the allocation $\left(n_{1}, n_{2}\right)$ they prefer. Parker and Van Alstyne (2005) use a slightly different approach, by directly assuming two-sided demands that depend on both prices: $n_{1}=D_{1}\left(p_{1}\right)+\alpha_{1} D_{2}\left(p_{2}\right)$ and $n_{2}=D_{2}\left(p_{2}\right)+\alpha_{2} D_{1}\left(p_{1}\right)$, where $0<\alpha_{1}, \alpha_{2}<1$.

Argenziano (2007) applies the global games methodology to the study of competition between one-sided networks. Each user receives a noisy signal regarding the standalone value of a given platform, but she is still assumed capable of calculating the optimal adoption strategies for all other users as a function of the platform price. Ambrus and Argenziano (2009) show that multiple asymmetric networks can coexist in equilibrium when agents are heterogeneous. In their model too, each individual agent observes all platform prices and calculates the resulting adoption decisions by all other agents. Thus, all of these papers rely on responsive expectations.

Passive expectations were first introduced in the economic literature on one-sided network effects by Katz and Shapiro (1985). This was also the first paper to explicitly distinguish passive expectations from responsive expectations. ${ }^{4}$ In particular, Katz and Shapiro (1985)

\footnotetext{
${ }^{4}$ Matutes and Vives (1996) make this distinction in a model of financial intermediation.
} 
study Cournot competition between $n$ firms (technologies) with direct network effects. In the main text of the paper, the authors analyze the case of passive expectations: each firm chooses its output taking other firms' decisions and users' expectations regarding firms' outputs as fixed. In the appendix, the authors also analyze the case of responsive expectations, where users' expectations adjust (correctly) based on firms' output decisions. They confirm that most of their analysis applies to both cases but do not compare firms' equilibrium profits and prices under the two types of expectations. The same Cournot model with passive and responsive expectations is also used by Economides (1996) to study the incentives of a network leader to invite entry of competing followers.

Gabszewicz and Wauthy (2012) is the only two-sided model we are aware of that incorporates passive expectations. Moreover, they investigate the difference in equilibrium outcomes between passive and responsive expectations. In their paper, users on both sides are differentiated by the intensity of their indirect network effects. With fixed rational expectations, their two-sided demands are $n_{i}=D_{i}\left(n_{j}^{e}, p_{i}\right)$, where $n_{j}^{e}$ is the demand on side $j$ expected by agents on side $i$. The platform maximizes $\Pi=p_{1} D_{1}\left(n_{2}^{e}, p_{1}\right)+p_{2} D_{2}\left(n_{1}^{e}, p_{2}\right)$ over $\left(p_{1}, p_{2}\right)$ treating $\left(n_{1}^{e}, n_{2}^{e}\right)$ as exogenously given. Then rational expectations require $\left(n_{1}, n_{2}\right)=\left(n_{1}^{e}, n_{2}^{e}\right)$, which closes the loop by determining equilibrium demands and prices. The same approach is used for competing platforms. Gabszewicz and Wauthy (2012) show that responsive expectations lead to wider participation on the platform. In our paper, we undertake a systematic investigation of how different levels of user information-leading to different types of expectations - affect the equilibrium outcome. Aside from passive and responsive expectations - investigated by Gabszewicz and Wauthy - we also analyze wary expectations and a hybrid model in which users with different expectation types are present in the market.

More recently, two papers have explicitly pointed out the impact of different user expectations (passive and responsive) on equilibrium allocations in markets with direct (i.e., one-sided) network effects. Griva and Vettas (2011) study price competition between two firms which are both horizontally and vertically differentiated. They find that competition under responsive expectations tends to be more intense and results in larger market shares captured by the high-quality firm, relative to the case with fixed expectations. Hurkens and Lopez (2012) study the impact of competition between communication networks. They show that replacing the standard assumption of responsive consumer expectations with a more realistic assumption of passive consumer expectations leads to radically different con- 
clusions regarding firm preferences for termination charges. Specifically, with responsive consumer expectations, firms prefer lower (below-cost) termination charges. Instead, with passive consumer expectations, firms prefer above cost termination charges, consistent with the real-world tension between mobile operators and their regulators.

There are three key differences between these two papers and our analysis. First, both papers rely on models with one-sided network effects, whereas our main model is two-sided. Second, they focus on duopoly settings only, whereas we are interested in comparing the impact of expectations in monopoly and duopoly contexts. Indeed, a key insight that emerges from our analysis is that the effect of user information about prices is very different depending on the market structure. Third, Griva and Vettas (2011) and Hurkens and Lopez (2012) only consider two extreme cases - all users holding passive expectations and all users holding responsive expectations. In contrast, we extend our baseline model to encompass expectation formation mechanisms in-between the extremes: a continuous hybrid of passive and responsive expectations, and wary expectations. Thus, we are able to study the effect of small changes in the nature of user expectations on market equilibria and firm profits.

Evans and Schmalensee (2010) study platform adoption in the presence of network effects with imperfect, dynamic adjustments of user participation decisions. At a high level, our paper is related to theirs in the effort to formally capture imperfections in the information held by users - a prevalent phenomenon in real-world settings. The key difference is that in their model, platform prices are fixed and the focus is on determining conditions (critical mass) under which the imperfect dynamic adjustment process converges to positive levels of platform adoption.

Finally, our analysis of user information about two-sided platform prices is related to the mechanisms of belief formation studied in the vertical contracting literature (Hart and Tirole 1990, McAfee and Schwartz 1994, Rey and Verge 2004). In that literature, a downstream firm D receiving an unexpected (out of equilibrium) offer from an upstream monopolist $U$ must form beliefs about the changes in U's offers to D's rivals (which D does not observe). Passive expectations in our context correspond to the notion of passive beliefs in the vertical contracting context: D does not adjust its beliefs about contracts offered to rivals when D receives an unexpected offer from $\mathrm{U}$. We also introduce the notion of wary expectations as a concept directly drawn from the notion of wary beliefs studied by McAfee and Schwartz (1994) and Rey and Verge (2004). In vertical contracting, D holds wary beliefs if, whenever it receives an unexpected offer, it anticipates that U also optimally adjusts its offers to D's 
rivals given the offer just received by D. In our context, a user who does not observe the price $p_{d}$ charged by a two-sided platform to developers holds wary expectations if, when presented with a price $p_{u}$, she assumes that the platform's price $p_{d}$ maximizes the platform's profits given $p_{u}$.

\section{Informed vs. Uninformed Users-Monopoly Plat- form}

Consider a monopoly two-sided platform. For convenience of reference, suppose it is a videogame console connecting users $(u)$ with game developers $(d)$. We assume linear demand on both sides:

$$
n_{u}=1+\alpha_{u} n_{d}^{e}-p_{u} \quad \text { and } \quad n_{d}=\alpha_{d} n_{u}^{e}-p_{d} .
$$

Thus, demand on each side depends positively on the expectation of participation on the other side: users expect $n_{d}^{e}$ developers to join and developers expect $n_{u}^{e}$ users to join. The surplus derived by a user from the participation of each developer is $\alpha_{u}>0$, while the profit made by each developer on every platform user is $\alpha_{d}>0$. We assume the following condition holds throughout the paper, which ensures all monopoly maximization problems are well-behaved: 5

$$
\alpha_{u}+\alpha_{d}<2
$$

In this formulation, the platform has standalone value normalized to 1 for every user but no standalone value for developers. Standalone values have no bearing on our results, therefore we have chosen the simplest possible formulation, with positive standalone value on one side $(u)$ only. This is also quite realistic given the videogame console example we have in mind: the console may offer first-party games, web browsing and streaming movie services to users, but writing games for a console with no users is arguably worthless for developers.

\subsection{Information and expectations}

Throughout the paper we assume that all developers are informed of all prices and therefore hold responsive expectations about user participation. In other words, their expectations

\footnotetext{
${ }^{5}$ This condition also implies $\alpha_{u} \alpha_{d}<1$.
} 
about user participation always match realized user participation:

$$
n_{u}^{e}=n_{u} \text { for any given price pair }\left(p_{u}, p_{d}\right)
$$

In contrast, we compare two polar cases on the user side:

(i) users are fully informed and therefore hold responsive expectations,

(ii) users are uninformed and hold passive expectations.

The asymmetry between users and developers in information about prices charged to the other side is realistic. Game developers are usually aware of console prices and have a good understanding of console user demand. On the other hand, most users have limited information about and understanding of the royalty arrangements between console manufacturers and game developers (or between Apple and iPhone app developers). These users most likely form their expectations of developer participation based on external information (e.g. news articles, word-of-mouth) and do not adjust them in response to the prices actually charged by platforms. ${ }^{6}$

\subsection{Informed users}

In this scenario, all users observe developer prices $p_{d}$ and adjust their expectations accordingly, so that $n_{d}^{e}$ matches realized developer participation $n_{d}$ for any price pair $\left(p_{u}, p_{d}\right)$ chosen by the platform. Two-sided demands from (1) can therefore be written:

$$
n_{u}=1+\alpha_{u} n_{d}-p_{u} \text { and } n_{d}=\alpha_{d} n_{u}-p_{d} .
$$

This is the standard formulation used in most of the two-sided market literature. It is straightforward to solve the last two equations for $\left(n_{u}, n_{d}\right)$ as functions of $\left(p_{u}, p_{d}\right)$ only, and then optimize the platform's profits $p_{u} n_{u}+p_{d} n_{d}$ directly over $\left(p_{u}, p_{d}\right)$. We obtain the following profit-maximizing prices and demands:

$$
p_{u}^{*}=\frac{2-\alpha_{d}\left(\alpha_{d}+\alpha_{u}\right)}{4-\left(\alpha_{d}+\alpha_{u}\right)^{2}} \quad \text { and } \quad p_{d}^{*}=\frac{\alpha_{d}-\alpha_{u}}{4-\left(\alpha_{d}+\alpha_{u}\right)^{2}}
$$

\footnotetext{
${ }^{6}$ Allowing both sides to be uninformed does not change our analysis in any meaningful way. All key results in the propositions below remain unchanged.
} 


$$
n_{u}^{*}=\frac{2}{4-\left(\alpha_{d}+\alpha_{u}\right)^{2}} \quad \text { and } \quad n_{d}^{*}=\frac{\alpha_{d}+\alpha_{u}}{4-\left(\alpha_{d}+\alpha_{u}\right)^{2}} .
$$

This leads to optimal platform profits:

$$
\Pi_{M}^{*}(\text { informed })=\frac{1}{4-\left(\alpha_{d}+\alpha_{u}\right)^{2}} .
$$

\subsection{Uninformed users}

In this scenario, users do not observe developer prices and do not adjust their expectations regarding developer participation $\left(n_{d}^{e}\right)$ in response to any changes in platform prices $\left(p_{d}\right.$ or $p_{u}$ ). In turn, the platform has no choice but to treat users' passive expectations $n_{d}^{e}$ as fixed when it sets its prices. Expectations are fulfilled in equilibrium. From the platform's perspective, two-sided realized demands now depend not just on prices but also on users' passive expectations $n_{d}^{e}$ :

$$
n_{u}=1+\alpha_{u} n_{d}^{e}-p_{u} \quad \text { and } \quad n_{d}=\alpha_{d}+\alpha_{u} \alpha_{d} n_{d}^{e}-p_{d}-\alpha_{d} p_{u}
$$

After optimizing the platform's profits $p_{u} n_{u}+p_{d} n_{d}$ over $\left(p_{u}, p_{d}\right)$, we obtain prices and realized demands $p_{u}^{*}\left(n_{d}^{e}\right), p_{d}^{*}\left(n_{d}^{e}\right), n_{u}^{*}\left(n_{d}^{e}\right)$ and $n_{d}^{*}\left(n_{d}^{e}\right)$, all of which depend on $n_{d}^{e}$. In equilibrium the rationality condition $n_{d}^{*}\left(n_{d}^{e}\right)=n_{d}^{e}$ must be satisfied. It directly leads to

$$
\begin{aligned}
& p_{u}^{*}=\frac{2-\alpha_{d}^{2}}{4-\left(\alpha_{d}+\alpha_{u}\right) \alpha_{d}} \quad \text { and } \quad p_{d}^{*}=\frac{\alpha_{d}}{4-\left(\alpha_{d}+\alpha_{u}\right) \alpha_{d}}, \\
& n_{u}^{*}=\frac{2}{4-\left(\alpha_{d}+\alpha_{u}\right) \alpha_{d}} \quad \text { and } \quad n_{d}^{*}=\frac{\alpha_{d}}{4-\left(\alpha_{d}+\alpha_{u}\right) \alpha_{d}} .
\end{aligned}
$$

The resulting optimal platform profits are

$$
\Pi_{M}^{*}(\text { uninformed })=\frac{4-\alpha_{d}^{2}}{\left(4-\left(\alpha_{d}+\alpha_{u}\right) \alpha_{d}\right)^{2}} .
$$

\subsection{Profit comparison and discussion}

We are interested in determining the effect of user expectations on platform profits. Comparing (4) and (7) leads to: 
Proposition 1 A monopoly platform earns higher profits when users are informed than when users are uninformed, i.e.,

$$
\Pi_{M}^{*}(\text { uninformed })<\Pi_{M}^{*}(\text { informed })
$$

This result is interpreted as follows. When all agents are informed, the platform is able to "commit" to the prices that maximize profits under rational expectations. Suppose now the price charged to developers is no longer observed by users, i.e. all users switch from being informed to being uninformed. Uninformed users (with passive expectations) do not adjust their expectations in response to changes in the developer price, therefore the platform is now tempted to increase the developer price above the level that was optimal with fully informed agents on both sides. But users anticipate this behavior ex-ante and adjust their expectations accordingly, which overall leads to lower platform profits. In other words, from the platform's perspective, facing uninformed users is akin to being unable to credibly announce (commit to) the optimal developer price to users. Conversely, uninformed users are in effect credibly committed to ignore the platform's price changes when forming their expectations. This leads the platform to charge ex-post a developer price that is too high for the platform's own good ex-ante. ${ }^{7}$

It is in fact possible to prove more generally (i.e., regardless of the shape of demand functions) that the case with fully informed users maximizes monopoly platform's profits across all settings with rational expectations.

Proposition 2 A monopoly two-sided platform achieves maximum profits in a rational expectations equilibrium when all agents on both sides are informed.

Proof. See Appendix A.1.

The logic behind this result is straightforward. Since expectations are fulfilled in equilibrium, the platform can replicate any rational expectations market allocation under the scenario with fully informed agents on both sides. However, this is not true if some users are not informed. In the presence of uninformed users, users' expectations are not fully re-

\footnotetext{
${ }^{7}$ We are grateful to an anonymous referee for suggesting this interpretation in terms of the ability to commit to prices.
} 
sponsive and therefore some rational expectations market allocations may not be attainable, which may prevent the platform from achieving the first-best outcome.

The result in Propositions 1 and 2 has two important implications. First, whenever feasible, a monopoly two-sided platform would like to inform all users of the prices charged to developers and of the way in which developer demand responds to price changes. ${ }^{8}$ As we will see in the next section, the opposite is true for competing platforms.

Second, from a social welfare standpoint, not only the platform, but also users and developers are better off when users are informed. Indeed, the expressions of equilibrium user and developer surplus are

$$
W_{u}=\frac{\left(1+\alpha_{u} n_{d}^{*}\right)}{4} \text { and } W_{d}=\frac{\left(1+\alpha_{u} n_{u}^{*}\right)}{4}
$$

Both surpluses are increasing in $\left(n_{u}^{*}, n_{d}^{*}\right)$, while user and developer demands are higher in the equilibrium with informed users than in the equilibrium with uninformed users (cf expressions (3) and (6)). Consequently, in the case of a platform monopolist, information disclosure is better for users, developers, the platform and, hence, for total social welfare. This means that in this case a policy that mandates information disclosure would be superfluous, since it is already in the best interest of the platform.

\section{Informed vs. Uninformed Users-Competing Plat- forms}

Let us now turn to the case of competition between two symmetric platforms. Users are distributed along a Hotelling segment $[0,1]$ with density 1 and transportation costs $t>0$. They are interested in joining at most one platform (i.e., they singlehome). From the perspective of developers, the two platforms are identical, but developers are allowed to multihome, i.e., join both platforms. ${ }^{9}$ Developers are differentiated by the fixed cost they incur when joining each platform. The fixed cost per platform is the same regardless of whether a developer joins one or both platforms, i.e., there are no economies of scope in joining multiple platforms.

\footnotetext{
${ }^{8}$ In this section we only compare the extreme cases where all users are informed or all users are uninformed. We study a model with heterogeneous users in Section 6.2.

${ }^{9}$ In the working paper version, we have also analyzed the corresponding model with singlehoming on both sides. The main result (ranking of profits under various types of expectations) is unchanged.
} 
User demands are:

$$
n_{u 1}=\frac{1}{2}+\frac{\alpha_{u}\left(n_{d 1}^{e}-n_{d 2}^{e}\right)+p_{u 2}-p_{u 1}}{2 t}
$$

for platform 1, and $n_{u 2}=1-n_{u 1}$ for platform 2, where $n_{d 1}^{e}$ and $n_{d 2}^{e}$ are users' expectations about developer participation on each platform. Developer demands are $n_{d 1}=\alpha_{d} n_{u 1}^{e}-p_{d 1}$ for platform 1 , and $n_{d 2}=\alpha_{d} n_{u 2}^{e}-p_{d 2}$ for platform 2 .

In order to guarantee all optimization problems with competing platforms are wellbehaved, we assume throughout the paper:

$$
t>\alpha_{u} \alpha_{d} .
$$

As in the previous section, all developers are informed and therefore hold responsive expectations about user participation, i.e., $n_{u i}^{e}=n_{u i}$ for $i=1,2$. Once again, we focus on the two polar cases on the user side: fully informed users and fully uninformed users.

\subsection{Informed users}

In this case, all users adjust their expectations $n_{d i}^{e}$ to match realized developer participation $n_{d i}(i=1,2)$ for any prices chosen by the two platforms. Two-sided demands are then:

$$
\begin{aligned}
& n_{u 1}=\frac{1}{2}+\frac{\alpha_{u}\left(n_{d 1}-n_{d 2}\right)+p_{u 2}-p_{u 1}}{2 t} \text { and } n_{d 1}=\alpha_{d} n_{u 1}-p_{d 1}, \\
& n_{u 2}=1-n_{u 1} \quad \text { and } \quad n_{d 2}=\alpha_{d} n_{u 2}-p_{d 2} .
\end{aligned}
$$

It is straightforward to solve for user and developer demands as functions of prices:

$$
\begin{aligned}
& n_{u 1}=\frac{1}{2}+\frac{p_{u 2}-p_{u 1}+\alpha_{u}\left(p_{d 2}-p_{d 1}\right)}{2\left(t-\alpha_{u} \alpha_{d}\right)} \\
& n_{d 1}=\frac{\alpha_{d}}{2}+\frac{\alpha_{d}\left(p_{u 2}-p_{u 1}\right)+\alpha_{d} \alpha_{u} p_{d 2}-\left(2 t-\alpha_{d} \alpha_{u}\right) p_{d 1}}{2\left(t-\alpha_{u} \alpha_{d}\right)} .
\end{aligned}
$$

Platforms simultaneously choose prices to maximize profits, $p_{u i} n_{u i}+p_{d i} n_{d i}$ for $i=1,2$. Taking the first-order conditions of platform $i$ 's profits in $p_{u i}$ and $p_{d i}$ and solving for the symmetric equilibrium, we obtain the following equilibrium prices and demands:

$$
p_{u}^{*}=t-\frac{3 \alpha_{d} \alpha_{u}}{4}-\frac{\alpha_{d}^{2}}{4} \quad \text { and } \quad p_{d}^{*}=\frac{\alpha_{d}-\alpha_{u}}{4},
$$




$$
n_{u}^{*}=\frac{1}{2} \quad \text { and } \quad n_{d}^{*}=\frac{\alpha_{d}+\alpha_{u}}{4}
$$

Equilibrium profits are:

$$
\Pi_{C}^{*}(\text { informed })=\frac{t}{2}-\frac{\alpha_{d}^{2}}{16}-\frac{6 \alpha_{d} \alpha_{u}+\alpha_{u}^{2}}{16}
$$

\subsection{Uninformed users}

In this scenario, users do not observe $\left(p_{d 1}, p_{d 2}\right)$ and do not adjust their expectations $n_{d i}^{e}$ to any changes in platforms' prices. Platform 1's profits are then simply:

$$
p_{u 1} n_{u 1}+p_{d 1} n_{d 1}=\left(p_{u 1}+\alpha_{d} p_{d 1}\right)\left(\frac{1}{2}+\frac{\alpha_{u}\left(n_{d 1}^{e}-n_{d 2}^{e}\right)+p_{u 2}-p_{u 1}}{2 t}\right)-p_{d 1}^{2}
$$

Taking the first order conditions in $\left(p_{u 1}, p_{d 1}\right)$ with fixed $\left(n_{d 1}^{e}, n_{d 2}^{e}\right)$ and imposing the symmetric equilibrium condition $n_{u 1}=n_{u 2}=\frac{1}{2}$ and the rational expectations condition $n_{d 1}=n_{d 2}=n_{d 1}^{e}=n_{d 2}^{e}$, we obtain the following equilibrium prices and demands:

$$
\begin{gathered}
p_{u}^{*}=t-\frac{\alpha_{d}^{2}}{4} \quad \text { and } \quad p_{d}^{*}=\frac{\alpha_{d}}{4}, \\
n_{u}^{*}=\frac{1}{2} \quad \text { and } \quad n_{d}^{*}=\frac{\alpha_{d}}{4},
\end{gathered}
$$

resulting in equilibrium profits:

$$
\Pi_{C}^{*}(\text { uninformed })=\frac{t}{2}-\frac{\alpha_{d}^{2}}{16}
$$

\subsection{Comparison and discussion}

Comparing equilibrium profit expressions (8) and (9) leads to:

Proposition 3 In the symmetric duopoly equilibrium with fixed Hotelling competition for users, platform profits are higher when all users are uninformed relative to the case when all users are informed:

$$
\Pi_{C}^{*}(\text { uninformed })>\Pi_{C}^{*}(\text { informed })
$$


Griva and Vettas (2011) obtain a similar result in a one-sided framework: they show that switching from passive to responsive expectations tends to lead to more intense competition in a duopoly with one-sided network effects.

Note that in Proposition 3 users are informed or uninformed at the industry level, i.e., they either know the developer prices for both platforms or for neither. ${ }^{10}$ Thus, the result in Proposition 3 implies that an industry association representing platform interests would prefer to minimize information released to users about developer prices. This stands in contrast to the case when the industry is monopolized by one platform - the latter always has an incentive to inform users (cf. Proposition 1).

The reason for this reversion of incentives to inform is as follows. When platforms compete for share in a market of fixed size on at least one side, each platform's incentives to lower price are strongest when users are informed of all prices. Indeed, such users respond to price decreases in two ways: they adjust their own demand, as well as their expectation of developer demand - both upwards. This creates intense price competition. With uninformed users, price competition is less severe because users completely ignore price cuts on the developer side and only consider the impact of a lower user price on their own participation instead of also factoring the impact of increased participation by users on developer demand.

Let us now consider the social welfare perspective. With competing platforms, as previously in the monopoly case, user and developer surpluses are increasing in equilibrium realized demands $\left(n_{u}^{*}, n_{d}^{*}\right)$ and these demands are (weakly) larger when users are informed. Thus, both users and developers prefer the scenario with uninformed users. This means that user and developer preferences are misaligned with the platforms' preferences. Consequently, a policy that mandates full information disclosure would help increase user and developer surplus, though it would hurt platforms' profits.

\section{Price comparison}

Like profits, the equilibrium pricing structures are different depending on whether users are informed or uninformed. Unlike profits, prices also have somewhat different comparative statics in the model parameters. This should not be too surprising: two-sided platforms

\footnotetext{
${ }^{10}$ The determination of the quilibrium in which each individual platform is able to choose whether or not to inform its users lies beyond the scope of this paper.
} 
typically re-adjust their two-dimensional pricing structures (e.g. increase one price and decrease the other) in response to changes in the external environment (e.g. the extent of information held by users).

Specifically, comparing monopoly platform prices (2) and (5), it is straightforward to show that:

$$
\begin{aligned}
& p_{u}^{*}(\text { informed }) \leq p_{u}^{*}(\text { uninformed }) \Longleftrightarrow \alpha_{u} \leq \alpha_{d} \\
& p_{d}^{*}(\text { informed }) \leq p_{d}^{*}(\text { uninformed }) \Longleftrightarrow \alpha_{d}\left(\alpha_{u}+\alpha_{d}\right)>2 .
\end{aligned}
$$

In both information scenarios, the respective equilibrium demands (3) and (6) are increasing in both network effects parameters $\alpha_{u}$ and $\alpha_{d}$, as expected. On the other hand, the comparative statics of equilibrium prices (2) and (5) in $\alpha_{u}$ and $\alpha_{d}$ are less trivial. We summarize them in the following table:

\begin{tabular}{l|c|c} 
& Monopoly user price $p_{u}^{*}$ & Monopoly developer price $p_{d}^{*}$ \\
\hline \multirow{4}{*}{$\begin{array}{l}\text { Informed } \\
\text { users }\end{array}$} & $\begin{array}{l}\text { negative iff } 2<\alpha_{d}\left(\alpha_{d}+\alpha_{u}\right) \\
\text { increasing in } \alpha_{u} \text { iff }\end{array}$ & $\begin{array}{l}\text { negative iff } \alpha_{d}<\alpha_{u} \\
\text { increasing in } \alpha_{d} \text { iff }\end{array}$ \\
& $4 \alpha_{u}>\alpha_{d}\left(\alpha_{d}+\alpha_{u}\right)^{2}$ & $\left(3 \alpha_{u}-\alpha_{d}\right)\left(\alpha_{u}+\alpha_{d}\right)<4$ \\
decreasing in $\alpha_{d}$ iff & decreasing in $\alpha_{u}$ iff \\
& $4 \alpha_{d}>\alpha_{u}\left(\alpha_{d}+\alpha_{u}\right)^{2}$ & $\left(3 \alpha_{d}-\alpha_{u}\right)\left(\alpha_{u}+\alpha_{d}\right)<4$ \\
\hline \multirow{3}{*}{$\begin{array}{l}\text { Uninformed } \\
\text { users }\end{array}$} & $\begin{array}{l}\text { negative iff } 2<\alpha_{d}^{2} \\
\text { increasing in } \alpha_{u} \text { iff } 2>\alpha_{d}^{2}\end{array}$ & always positive \\
& decreasing in $\alpha_{d}$ iff & always increasing in $\alpha_{d}$ \\
& $4 \alpha_{d}>\alpha_{u}\left(2+\alpha_{d}^{2}\right)$ & always increasing in $\alpha_{u}$
\end{tabular}

Turning now to competing platform prices, we have:

$$
\begin{aligned}
& p_{u}^{*} \text { (informed) }<p_{u}^{*} \text { (uninformed) for all } \alpha_{u}, \alpha_{d}, \\
& p_{d}^{*} \text { (informed) }<p_{d}^{*} \text { (uninformed) for all } \alpha_{u}, \alpha_{d} .
\end{aligned}
$$

and the differences in comparative statics are summarized in the following table: 


\begin{tabular}{l|l|l} 
& \multicolumn{1}{|c|}{ Duopoly user price $p_{u}^{*}$} & \multicolumn{1}{c}{ Duopoly developer price $p_{d}^{*}$} \\
\hline Informed & negative iff $t<\left(3 \alpha_{d} \alpha_{u}+\alpha_{d}^{2}\right) / 4$ & negative iff $\alpha_{d}<\alpha_{u}$ \\
users & decreasing in $\alpha_{u}$ and $\alpha_{d}$ & increasing in $\alpha_{d}$; decreasing in $\alpha_{u}$ \\
\hline Uninformed & negative iff $t<\alpha_{d}^{2} / 4$ & always positive \\
users & decreasing in $\alpha_{d}$; constant in $\alpha_{u}$ & increasing in $\alpha_{d}$; constant in $\alpha_{u}$
\end{tabular}

These differences can have significant empirical implications. For instance, a monopoly two-sided platform facing uninformed users never subsidizes developers, but it might do so when users are informed (when $\alpha_{u}>\alpha_{d}$ ). Again, this is because uninformed users are less responsive to price cuts, so there is less to be gained from lowering prices on either side. Furthermore, different comparative statics in $\left(\alpha_{u}, \alpha_{d}\right)$ imply that equilibrium prices respond differently to changes in the network effects parameters under different information scenarios. Thus, if one were to estimate network effects parameters based on observed prices, results would be different depending on the level of user information.

\section{Extensions}

In this section, we briefly discuss three extensions, which provide robustness checks to our simple model from the previous sections.

\subsection{Wary expectations}

When users are uninformed, they cannot revise their expectations regarding developer participation based on changes in developer prices (which they do not observe). However, users may adjust expectations based on changes in user prices (which they observe). Accordingly, in this section we study an alternative to the passive expectations studied above by assuming uninformed users hold wary expectations. Our concept of wary expectations is directly adapted from the concept of wary beliefs from the vertical contracting literature (McAfee and Schwartz (1994), Rey and Verge (2004)). An uninformed user who does not observe developer prices is said to hold wary expectations if, when presented with any off-equilibrium price $p_{u}$, she assumes that the platform has also adjusted its price to developers, $p_{d}$, to maximize profits, given $p_{u}$. This leads the user to form her expectation of the number of developers who join the platform based on the price that she observes: $n_{d}^{e}\left(p_{u}\right)$. 
The main difference relative to passive expectations is that wary users attempt to make the optimal inference about developer prices, based on the information available to them, i.e. $p_{u}$ and the structure of developer demand. Thus, wary expectations can be viewed as lying somewhere in-between the two polar extremes of perfectly responsive and perfectly passive expectations in terms of user information and responsiveness to platform prices.

Consistent with this interpretation, in Appendix A.2, we show that platform profits when all users are uninformed and hold wary expectations are in-between profits with fully informed users and profits with fully uninformed users holding passive expectations. This result holds both with a monopoly and with competing platforms. In other words:

$$
\left.\Pi_{M}^{*}(\text { informed })>\Pi_{M}^{*} \text { (uninformed-wary) }>\Pi_{M}^{*} \text { (uninformed-passive }\right)
$$

and $\quad \Pi_{C}^{*}$ (uninformed-passive $)>\Pi_{C}^{*}$ (uninformed-wary) $>\Pi_{C}^{*}$ (informed).

These results confirm the main insights derived in Sections 3 and 4. A monopoly platform prefers to face more informed users, where one can view uninformed wary users as being more informed than uninformed passive users. Conversely, competing platforms prefer to face less informed users. In both cases, the explanation is similar to the one provided in Sections 3 and 4: user information leads to more price responsiveness, which is good for a monopolist but bad for competing platforms in a market of fixed size.

\subsection{Heterogeneous users}

A different way of modelling intermediate levels of user information is to consider a hybrid scenario in which some users are informed, while others are uninformed and hold passive expectations. Indeed, up to now we have only considered "pure" forms of user information and expectations, i.e. scenarios in which all users held the same amount of information and the same type of expectation. We are particularly interested in seeing how equilibrium platform profits respond to small changes in the level of information held by users.

Specifically, assume that a fraction $\lambda$ of users are informed, while the remaining fraction $(1-\lambda)$ of users are uninformed and hold passive expectations. All developers are informed as before. We have studied the extreme cases $\lambda=1$ (all users are informed) and $\lambda=0$ (all users are uninformed and passive) in Sections 3 and 4. 
In Appendix A.3 we show that the optimal profits for a monopoly platform are

$$
\Pi_{M}^{*}(\lambda)=\frac{4-\left(\alpha_{d}+\lambda \alpha_{u}\right)^{2}}{\left(4-\left(\alpha_{d}+\alpha_{u}\right)\left(\alpha_{d}+\lambda \alpha_{u}\right)\right)^{2}}
$$

while in Appendix A.4 we show that the equilibrium platform profits in the Hotelling duopoly from Section 4 are

$$
\Pi_{C}^{*}(\lambda)=\frac{t}{2}-\frac{\alpha_{d}^{2}}{16}-\frac{6 \alpha_{d} \alpha_{u} \lambda+\alpha_{u}^{2} \lambda^{2}}{16}
$$

Thus, as one might expect based on the results in Propositions 1 and $3, \Pi_{M}^{*}(\lambda)$ is increasing in $\lambda$, while $\Pi_{C}^{*}(\lambda)$ is decreasing. In other words, a monopoly platform is always better off when it faces more informed users. By contrast, platforms competing for share in a market of fixed size on at least one side are worse off when there are more informed users.

\subsection{Generalized competition}

Proposition 3 implies that competing platforms with fixed market size on at least one side are better off when users are uninformed about developer prices - the opposite implication than for a monopoly platform (Propositions 1 and 2). In Appendix A.4, we use a more general model of platform competition to show that equilibrium profits are larger with uninformed users than with informed users if and only if competition for users is sufficiently intense. Figure 1 below illustrates this result. In this model, the intensity of competition is measured by a parameter $x$, which varies between $x=0$ (each platform is a monopolist as in Section 3 ) to $x=1$ (Hotelling competition with fixed market size as in Section 4).

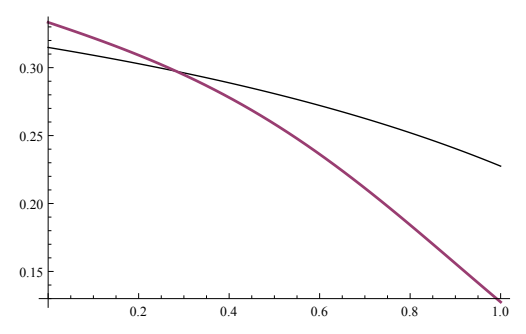

Figure 1: Platform profits with fully informed (thick line) and fully uninformed users (thin line), both as functions of $x$, with parameter values $t=0.5, \alpha_{u}=0.4$ and $\alpha_{d}=0.6$.

One can then combine generalized competition (intensity of competition $x$ ) with heterogeneous user information (fraction of informed users $\lambda$ ). We explicitly solve this model and 
derive equilibrium platform profits $\Pi_{C}^{*}(x, \lambda)$ in Appendix A.4. Here let us simply emphasize the following points. When $x=1$, the model is equivalent to the competition scenario with fixed user market size: equilibrium platform profits are decreasing in $\lambda$ (cf. Section 6.2). When $x=0$, the model is equivalent to two independent two-sided monopolies, so that equilibrium profits are increasing in $\lambda$. As $x$ increases from 0 to 1 , equilibrium profits $\Pi_{C}^{*}(x, \lambda)$ are first increasing, then single-peaked, and lastly decreasing in $\lambda$. This is illustrated in Figure 2 .

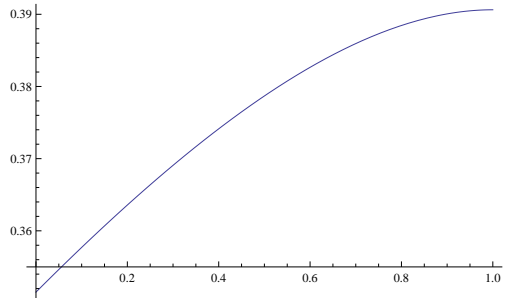

(a) $\Pi_{C}^{*}(0, \lambda)$

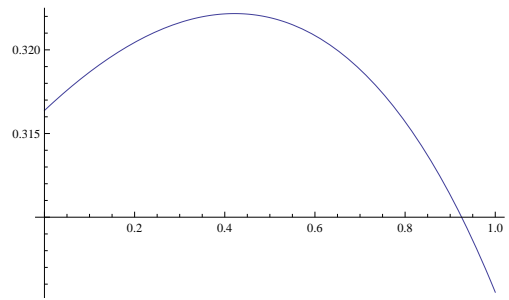

(b) $\Pi_{C}^{*}(0.35, \lambda)$

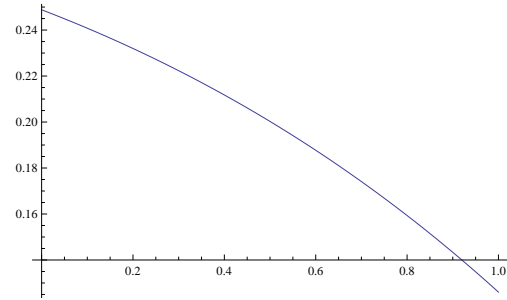

(c) $\Pi_{C}^{*}(0.8, \lambda)$

Figure 2: Competing platform profits $\left(\Pi_{C}^{*}(x, \lambda)\right)$, as a function of $\lambda$, for three different values of $x$. All graphs have parameters $t=0.5, \alpha_{u}=0.5, \alpha_{d}=0.7$.

Thus, for general $x \in(0,1)$, the fraction of informed users $\lambda$ has two conflicting effects: user market expansion through the Hotelling hinterlands tends to make increases in $\lambda$ desirable for the two platforms, whereas competition for users on the interior of the Hotelling segment tends to make increases in $\lambda$ undesirable (increased competitive pressure).

In other words, platforms benefit from increased information in the market if competition is not too intense. As competitive pressure increases, information starts having a negative effect on platform profits. This is because the degree of information available in the market (captured by $\lambda$ and taken as exogenously given) and the intensity of platform competition (captured by $x$ and also taken as exogenously given) tend to be substitutes from the perspective of platform profits.

\section{Conclusions}

This paper has explored how equilibrium outcomes in a two-sided platform market are affected by different levels of user information about prices charged to the other side. 
We have shown that the level of user information affects platform profits differently depending on market structure. Broadly speaking, platforms with more market power prefer facing more informed users. Platforms that compete in a market of fixed size have the opposite preference: they derive higher profits when users are less informed and therefore have less responsive expectations. The main reason is that information about prices amplifies the effect of price reductions. For platforms with market power, this is good news because they can capture the demand increases and achieve higher profits. For competing platforms, more information is typically bad news because it intensifies price competition.

From a strategy perspective, these results imply that platforms have incentives to affect the information held by users about prices charged to the other side of the market. A monopoly platform would always wish to inform and educate users about developer prices and the structure of developer demand. Platforms facing intense competition wish to minimize the information provided to users. Finally, platforms facing competition of moderate intensity may pursue an interior, ideal level of information to be provided (cf. Section 6.3).

Finally, from a policy perspective, our results imply that mandating full information disclosure by platforms leads to an increase in user as well as developer surplus in the case of competing platforms with fixed market size. Indeed, in that scenario, mandating information disclosure unambiguously leads to lower prices on both sides of the market and higher developer demand (see price and demand expressions in Section 4).

In the case of a monopoly platform, mandating information disclosure leads to higher demands on both sides (see expressions (3) and (6)), which implies that surplus on both sides is also higher under full information (even though prices may be higher or lower, as discussed in Section 5). Here, however, mandating information disclosure is superfluous because it is also in the best interest of the platform. On the other hand, with competing platforms, the policy maker has to weigh the interests of users and developers against those of the platforms.

\section{A Appendix: Proofs}

\section{A.1 Proof of proposition 2}

Let two-sided demands be $D_{u}\left(n_{d}^{e}, p_{u}\right)$ and $D_{d}\left(n_{u}^{e}, p_{d}\right)$ and define the corresponding inverse demand 
functions $\widetilde{D}_{u}\left(p_{u}, p_{d}\right)$ and $\widetilde{D}_{d}\left(p_{u}, p_{d}\right)$ as the solutions $\left(n_{u}, n_{d}\right)$ to the system of two equations:

$$
n_{u}=D_{u}\left(n_{d}, p_{u}\right) \quad \text { and } \quad n_{d}=D_{d}\left(n_{u}, p_{d}\right)
$$

Denote by $\left(p_{u}^{*}, p_{d}^{*}\right)$ the profit-maximizing prices when all agents on both sides are informed and therefore hold responsive expectations:

$$
\left(p_{u}^{*}, p_{d}^{*}\right)=\arg \max _{p_{u}, p_{d}}\left\{p_{u} \widetilde{D}_{u}\left(p_{u}, p_{d}\right)+p_{d} \widetilde{D}_{d}\left(p_{u}, p_{d}\right)\right\}
$$

Consider now some other rational expectations equilibrium in which the platform's profitmaximizing prices are $\widehat{p}_{u}$ and $\widehat{p}_{d}$ and resulting two-sided demands are $\widehat{n}_{u}$ and $\widehat{n}_{d}$ (for example, this could be the profit-maximizing equilibrium when a fraction of agents on each side are uninformed and hold passive expectations). Since expectations are fulfilled at the equilibrium prices, we must have:

$$
\widehat{n}_{u}=D_{u}\left(\widehat{n}_{d}, \widehat{p}_{u}\right) \quad \text { and } \quad \widehat{n}_{d}=D_{d}\left(\widehat{n}_{u}, \widehat{p}_{d}\right)
$$

so that:

$$
\widehat{n}_{u}=\widetilde{D}_{u}\left(\widehat{p}_{u}, \widehat{p}_{d}\right) \quad \text { and } \quad \widehat{n}_{d}=\widetilde{D}_{d}\left(\widehat{p}_{u}, \widehat{p}_{d}\right)
$$

which means that platform profits in this equilibrium are equal to $\widehat{p}_{u} \widetilde{D}_{u}\left(\widehat{p}_{u}, \widehat{p}_{d}\right)+\widehat{p}_{d} \widetilde{D}_{d}\left(\widehat{p}_{u}, \widehat{p}_{d}\right)$. But by definition, $\left(p_{u}^{*}, p_{d}^{*}\right)$ maximizes $p_{u} \widetilde{D}_{u}\left(p_{u}, p_{d}\right)+p_{d} \widetilde{D}_{d}\left(p_{u}, p_{d}\right)$ over the $\left(p_{u}, p_{d}\right)$ space, therefore the platform's optimal profits are always highest with fully informed agents on both sides.

The intuition for this result is straightforward. Since expectations are fulfilled in equilibrium, the platform can replicate any rational expectations market allocation $\left(\widehat{p}_{u}, \widehat{p}_{d}, \widehat{n}_{u}, \widehat{n}_{d}\right)$ under full information on both sides, simply by charging $\left(\widehat{p}_{u}, \widehat{p}_{d}\right)$. But by definition it can do at least as well in terms of profits by charging $\left(p_{u}^{*}, p_{d}^{*}\right)$.

\section{A.2 Uninformed users with wary expectations}

\section{A.2.1 Monopoly}

When all users are uninformed and hold wary expectations, two-sided demands are:

$$
n_{u}=1+\alpha_{u} n_{d}^{e}\left(p_{u}\right)-p_{u} \quad \text { and } \quad n_{d}=\alpha_{d} n_{u}-p_{d}
$$

where $n_{d}^{e}\left(p_{u}\right)$ is the wary expectation formed by users about the number of participating developers. 
In calculating $n_{d}^{e}\left(p_{u}\right)$, users assume the platform sets $p_{d}$ optimally given $p_{u}$.

Denote by $p_{d}^{e}\left(p_{u}\right)$ the price that users with wary expectations anticipate the platform is charging. Thus, $p_{d}^{e}\left(p_{u}\right)$ and $n_{d}^{e}\left(p_{u}\right)$ are determined by maximizing profits $p_{u} n_{u}+p_{d} n_{d}$ over $p_{d}$ (with $p_{u}$ and $n_{d}^{e}$ taken as exogenously given), and then imposing $n_{d}=n_{d}^{e}$. In other words, when users form wary expectations, they take into account that the platform cannot influence their expectations once $p_{u}$ is fixed, but they impose rationality of those expectations, i.e., the developer demand resulting from the platform's hypothetical optimization must be consistent with (equal to) users' expectations.

The platform's profits can be expressed as:

$$
p_{u} n_{u}+p_{d} n_{d}=\left(p_{u}+\alpha_{d} p_{d}\right)\left[1+\alpha_{u} n_{d}^{e}-p_{u}\right]-p_{d}^{2}
$$

Taking the first-order condition in $p_{d}$ and imposing $n_{d}=n_{d}^{e}$, we obtain:

$$
n_{d}^{e}\left(p_{u}\right)=p_{d}^{e}\left(p_{u}\right)=\frac{\alpha_{d}\left(1-p_{u}\right)}{2-\alpha_{u} \alpha_{d}} .
$$

With the expression of user wary expectations in hand, we can now turn to the actual optimization of platform profits:

$$
p_{u} n_{u}+p_{d} n_{d}=\frac{2\left(p_{u}+\alpha_{d} p_{d}\right)\left(1-p_{u}\right)}{2-\alpha_{u} \alpha_{d}}-p_{d}^{2}
$$

which the platform maximizes over $\left(p_{u}, p_{d}\right)$. It is straightforward to obtain:

$$
\begin{aligned}
p_{u}^{*} & =\frac{2-\alpha_{u} \alpha_{d}-\alpha_{d}^{2}}{4-2 \alpha_{u} \alpha_{d}-\alpha_{d}^{2}} \quad \text { and } \quad p_{d}^{*}=\frac{\alpha_{d}}{4-2 \alpha_{u} \alpha_{d}-\alpha_{d}^{2}}, \\
n_{u}^{*} & =\frac{2}{4-2 \alpha_{u} \alpha_{d}-\alpha_{d}^{2}} \quad \text { and } \quad n_{d}^{*}=\frac{\alpha_{d}}{4-2 \alpha_{u} \alpha_{d}-\alpha_{d}^{2}} .
\end{aligned}
$$

Note in particular that $n_{d}^{*}=n_{d}^{e}\left(p_{u}^{*}\right)$, i.e. wary expectations are rational, as noted from the outset. The resulting platform profits are:

$$
\Pi_{M}^{*}(\text { uninformed-wary })=\frac{1}{4-2 \alpha_{u} \alpha_{d}-\alpha_{d}^{2}} .
$$

Comparing (14) with (4) and (7) yields:

$$
\Pi_{M}^{*} \text { (informed) }>\Pi_{M}^{*} \text { (uninformed-wary) }>\Pi_{M}^{*} \text { (uninformed-passive) } .
$$




\section{A.2.2 Competition}

Let us now turn to the Hotelling duopoly case, the same as the one studied in Section 4, except that now users are uninformed and hold wary expectations. Specifically, users form wary expectations $n_{d 1}^{e}$ and $n_{d 2}^{e}$ by assuming the two platforms' developer prices $p_{d 1}$ and $p_{d 2}$ are set optimally (Nash equilibrium) given $\left(p_{u 1}, p_{u 2}\right)$ and $\left(n_{d 1}^{e}, n_{d 2}^{e}\right)$. The expectations $n_{d 1}^{e}$ and $n_{d 2}^{e}$, which depend on $p_{u 1}$ and $p_{u 2}$, are determined by optimizing platform 1 's profits over $p_{d 1}$ :

$$
p_{u 1} n_{u 1}+p_{d 1} n_{d 1}=\left(p_{u 1}+\alpha_{d} p_{d 1}\right)\left[\frac{1}{2}+\frac{\alpha_{u}\left(n_{d 1}^{e}-n_{d 2}^{e}\right)+p_{u 2}-p_{u 1}}{2 t}\right]-p_{d 1}^{2}
$$

Taking the first-order condition in $p_{d 1}$ (this is the platform optimization problem that users assume is taking place) yields:

$$
p_{d 1}^{e}=\frac{\alpha_{d}}{4}+\frac{\alpha_{u} \alpha_{d}\left(n_{d 1}^{e}-n_{d 2}^{e}\right)+\alpha_{d}\left(p_{u 2}-p_{u 1}\right)}{4 t} .
$$

Similarly for platform 2 and $p_{d 2}^{e}$.

At these prices:

$$
n_{d 1}-n_{d 2}=\alpha_{d}\left(n_{u 1}-n_{u 2}\right)-\left(p_{d 1}^{e}-p_{d 2}^{e}\right)=\frac{\alpha_{d} \alpha_{u}\left(n_{d 1}^{e}-n_{d 2}^{e}\right)+\alpha_{d}\left(p_{u 2}-p_{u 1}\right)}{2 t} .
$$

Finally, imposing the rationality condition $n_{d 1}=n_{d 1}^{e}$ and $n_{d 2}=n_{d 2}^{e}$, we obtain:

$$
n_{d 1}^{e}-n_{d 2}^{e}=p_{d 1}^{e}-p_{d 2}^{e}=\frac{\alpha_{d}\left(p_{u 2}-p_{u 1}\right)}{2 t-\alpha_{d} \alpha_{u}}
$$

Using the last equation, we can express user demand as a function of prices only:

$$
n_{u 1}=\frac{1}{2}+\frac{p_{u 2}-p_{u 1}}{2 t-\alpha_{u} \alpha_{d}} .
$$

We can now write platform 1's profits as:

$$
p_{u 1} n_{u 1}+p_{d 1} n_{d 1}=\left(p_{u 1}+\alpha_{d} p_{d 1}\right)\left(\frac{1}{2}+\frac{p_{u 2}-p_{u 1}}{2 t-\alpha_{u} \alpha_{d}}\right)-p_{d 1}^{2}
$$

Taking the first-order conditions in $p_{u 1}$ and $p_{u 2}$ and imposing the condition for a symmetric equilibrium, we obtain:

$$
p_{u}^{*}=t-\frac{\alpha_{d} \alpha_{u}}{2}-\frac{\alpha_{d}^{2}}{4} \quad \text { and } \quad p_{d}^{*}=\frac{\alpha_{d}}{4},
$$




$$
n_{u}^{*}=\frac{1}{2} \quad \text { and } \quad n_{d}^{*}=\frac{\alpha_{d}}{4} .
$$

leading to equilibrium profits:

$$
\Pi_{C}^{*}(\text { uninformed-wary })=\frac{t}{2}-\frac{\alpha_{d} \alpha_{u}}{4}-\frac{\alpha_{d}^{2}}{16}
$$

Comparing (15) with (8) and (9) yields:

$$
\Pi_{C}^{*}(\text { uninformed-passive })>\Pi_{C}^{*}(\text { uninformed-wary })>\Pi_{C}^{*}(\text { informed })
$$

\section{A.3 Heterogeneous users-Monopoly}

Here, we derive the expressions of optimal prices, demands and profits for a monopoly platform, when a proportion $\lambda$ of users is informed and a fraction $(1-\lambda)$ is uninformed and holds passive expectations $n_{d}^{e}$. Two-sided demands are:

$$
\begin{gathered}
n_{u}=\lambda\left(1+\alpha_{u} n_{d}-p_{u}\right)+(1-\lambda)\left(1+\alpha_{u} n_{d}^{e}-p_{u}\right) \\
n_{d}=\alpha_{d} n_{u}-p_{d} .
\end{gathered}
$$

Solving for realized demands $\left(n_{u}, n_{d}\right)$ as functions of prices $\left(p_{u}, p_{d}\right)$ and fixed expectations $n_{d}^{e}$ we obtain:

$$
\begin{gathered}
n_{u}=\frac{1+\alpha_{u}(1-\lambda) n_{d}^{e}-p_{u}-\alpha_{u} \lambda p_{d}}{1-\alpha_{u} \alpha_{d} \lambda} \\
n_{d}=\frac{\alpha_{d}+\alpha_{u} \alpha_{d}(1-\lambda) n_{d}^{e}-\alpha_{d} p_{u}-p_{d}}{1-\alpha_{u} \alpha_{d} \lambda} .
\end{gathered}
$$

Plugging these two expressions in the expression of platform profits $\Pi=p_{u} n_{u}+p_{d} n_{d}$, taking the two first order conditions in $\left(p_{u}, p_{d}\right)$ and imposing the rational expectations condition $n_{d}^{*}=n_{d}^{e}$ in equilibrium, we obtain:

$$
\begin{aligned}
n_{u}^{*}-\frac{p_{u}^{*}+\alpha_{d} p_{d}^{*}}{1-\alpha_{u} \alpha_{d} \lambda} & =0, \\
n_{d}^{*}-\frac{\alpha_{u} \lambda p_{u}^{*}+p_{d}^{*}}{1-\alpha_{u} \alpha_{d} \lambda} & =0 .
\end{aligned}
$$

Furthermore, evaluating the expressions of $\left(n_{u}, n_{d}\right)$ as functions of $\left(p_{u}, p_{d}, n_{d}^{e}\right)$ in equilibrium, 
we also have:

$$
\begin{gathered}
\left(1-\alpha_{u} \alpha_{d} \lambda\right) n_{u}^{*}-\alpha_{u}(1-\lambda) n_{d}^{*}=1-p_{u}^{*}-\alpha_{u} \lambda p_{d}^{*} \\
\left(1-\alpha_{u} \alpha_{d}\right) n_{d}^{*}=\alpha_{d}-\alpha_{d} p_{u}^{*}-p_{d}^{*}
\end{gathered}
$$

We can then solve the last 4 equations for the equilibrium prices and demands, obtaining:

$$
\begin{aligned}
& p_{u}^{*}=\frac{2-\alpha_{d}\left(\alpha_{d}+\alpha_{u} \lambda\right)}{4-\left(\alpha_{u}+\alpha_{d}\right)\left(\alpha_{d}+\alpha_{u} \lambda\right)} \quad \text { and } \quad p_{d}^{*}=\frac{\alpha_{d}-\alpha_{u} \lambda}{4-\left(\alpha_{u}+\alpha_{d}\right)\left(\alpha_{d}+\alpha_{u} \lambda\right)}, \\
& n_{u}^{*}=\frac{2}{4-\left(\alpha_{u}+\alpha_{d}\right)\left(\alpha_{d}+\alpha_{u} \lambda\right)} \quad \text { and } \quad n_{d}^{*}=\frac{\alpha_{d}+\alpha_{u} \lambda}{4-\left(\alpha_{u}+\alpha_{d}\right)\left(\alpha_{d}+\alpha_{u} \lambda\right)} .
\end{aligned}
$$

Finally, equilibrium monopoly profits are:

$$
\Pi_{M}^{*}(\lambda)=\frac{4-\left(\alpha_{d}+\alpha_{u} \lambda\right)^{2}}{\left(4-\left(\alpha_{u}+\alpha_{d}\right)\left(\alpha_{d}+\alpha_{u} \lambda\right)\right)^{2}} .
$$

Setting $\lambda=1$ we obtain (2), (3) and (4). Setting $\lambda=0$, we obtain (5), (6) and (7).

\section{A.4 Heterogeneous users-Generalized competition}

Here, we directly treat the general duopoly model with heterogeneous users. The user market is composed of three segments: the Hotelling segment of density $x$ and two symmetric "hinterlands" of density $(1-x)$, on which user demand is identical to the demand for a monopoly platform from Section 3. A fraction $\lambda$ of users is informed, while a fraction $(1-\lambda)$ is uninformed and holds passive expectations. The distribution $(\lambda, 1-\lambda)$ is independent of the position of a given user on the Hotelling line with hinterlands. The structure of developer demand remains unchanged, i.e. each platform acts as a monopolist vis-a-vis developers.

User demand for platform 1 is:

$$
\begin{aligned}
n_{u 1}= & \lambda\left(x\left(\frac{1}{2}+\frac{\alpha_{u}\left(n_{d 1}-n_{d 2}\right)-\left(p_{u 1}-p_{u 2}\right)}{2 t}\right)+(1-x)\left(1+\alpha_{u} n_{d 1}-p_{u 1}\right)\right) \\
& +(1-\lambda)\left(x\left(\frac{1}{2}+\frac{\alpha_{u}\left(n_{d 1}^{e}-n_{d 2}^{e}\right)-\left(p_{u 1}-p_{u 2}\right)}{2 t}\right)+(1-x)\left(1+\alpha_{u} n_{d 1}^{e}-p_{u 1}\right)\right) \\
= & 1-\frac{x}{2}+x\left(\frac{\alpha_{u}\left(\lambda n_{d 1}+(1-\lambda) n_{d 1}^{e}-\lambda n_{d 2}-(1-\lambda) n_{d 2}^{e}\right)-\left(p_{u 1}-p_{u 2}\right)}{2 t}\right) \\
& +(1-x)\left(\alpha_{u}\left(\lambda n_{d 1}+(1-\lambda) n_{d 1}^{e}\right)-p_{u 1}\right)
\end{aligned}
$$


while developer demand for platform 1 is:

$$
n_{d 1}=\alpha_{d} n_{u 1}-p_{d 1}
$$

and symmetrically for platform 2 .

Note that $\lambda=0$ corresponds to the generalized duopoly scenario discussed at the end of Section 4 , while $x=0$ and $x=1$ correspond to the hybrid scenarios studied in Section 6.2.

To determine the pricing equilibrium, we need first to determine demands as functions of prices and constants (including passive expectations held by a fraction $(1-\lambda)$ of users). To do that, we first determine (using $\left.n_{d 1} \pm n_{d 2}=\alpha_{d}\left(n_{u 1} \pm n_{u 2}\right)-\left(p_{d 1} \pm p_{d 2}\right)\right)$ :

$$
\begin{aligned}
n_{u 1}+n_{u 2} & =\frac{2-x+(1-x)\left((1-\lambda) \alpha_{u}\left(n_{d 1}^{e}+n_{d 2}^{e}\right)-\left(p_{u 1}+p_{u 2}\right)-\lambda \alpha_{u}\left(p_{d 1}+p_{d 2}\right)\right)}{1-(1-x) \lambda \alpha_{u} \alpha_{d}} \\
n_{u 1}-n_{u 2} & =\frac{(x+t(1-x))\left((1-\lambda) \alpha_{u}\left(n_{d 1}^{e}-n_{d 2}^{e}\right)-\left(p_{u 1}-p_{u 2}\right)-\lambda \alpha_{u}\left(p_{d 1}-p_{d 2}\right)\right)}{t-[x+t(1-x)] \lambda \alpha_{u} \alpha_{d}}
\end{aligned}
$$

Platform 1's profits are $p_{u 1} n_{u 1}+p_{d 1} n_{d 1}$, which can be re-written $\left(p_{u 1}+\alpha_{d} p_{d 1}\right) n_{u 1}-p_{d 1}^{2}$. In this expression, $n_{u 1}$ is obtained by adding (16) and (17) and dividing by 2 . In the symmetric equilibrium, expectations by uninformed users are fulfilled. Taking the first order conditions of the profit function in $p_{u 1}$ and $p_{d 1}$ and evaluating at equilibrium values, we obtain:

$$
\begin{gathered}
n_{u}^{*}=A(x, \lambda)\left(p_{u}^{*}+\alpha_{d} p_{d}^{*}\right), \\
n_{u}^{*}=B(\lambda) p_{d}^{*}
\end{gathered}
$$

where we have denoted:

$$
A(x, \lambda) \equiv \frac{1}{2}\left(\frac{1-x}{1-(1-x) \lambda \alpha_{u} \alpha_{d}}+\frac{x+t(1-x)}{t-[x+t(1-x)] \lambda \alpha_{u} \alpha_{d}}\right) \quad \text { and } \quad B(\lambda) \equiv \frac{2}{\alpha_{d}-\lambda \alpha_{u}} .
$$

From the initial demand functions and symmetry, we have:

$$
n_{u}^{*}=C(x)-D(x)\left(p_{u}^{*}+\alpha_{u} p_{d}^{*}\right) \quad \text { and } \quad n_{d}^{*}=\alpha_{d} n_{u}^{*}-p_{d}^{*}
$$

where:

$$
C(x) \equiv \frac{1-x / 2}{1-(1-x) \alpha_{u} \alpha_{d}} \quad \text { and } \quad D(x) \equiv \frac{1-x}{1-(1-x) \alpha_{u} \alpha_{d}}
$$


It is then straightforward to solve (18), (19) and (20) in order to determine the equilibrium prices and demands:

$$
\begin{gathered}
p_{u}^{*}=\frac{C(x)\left(B(\lambda)-\alpha_{d} A(x, \lambda)\right)}{\Delta(x, \lambda)} \text { and } p_{d}^{*}=\frac{C(x) A(x, \lambda)}{\Delta(x, \lambda)}, \\
n_{u}^{*}=\frac{C(x) A(x, \lambda) B(\lambda)}{\Delta(x, \lambda)} \text { and } n_{d}^{*}=\frac{\left(\alpha_{d} B(\lambda)-1\right) C(x) A(x, \lambda)}{\Delta(x, \lambda)},
\end{gathered}
$$

where:

$$
\Delta(x, \lambda) \equiv A(x, \lambda) B(\lambda)+\left(\alpha_{u}-\alpha_{d}\right) D(x) A(x, \lambda)+D(x) B(\lambda) .
$$

Finally, equilibrium platform profits are:

$$
\Pi_{C}^{*}(x, \lambda)=\frac{C(x)^{2} A(x, \lambda)\left[B(\lambda)^{2}-A(x, \lambda)\right]}{\Delta(x, \lambda)^{2}} .
$$

It is easily verified that:

- $A(0, \lambda)=\frac{1}{1-\lambda \alpha_{u} \alpha_{d}}, C(0)=D(0)=\frac{1}{1-\alpha_{u} \alpha_{d}}$ and $\Delta(0, \lambda)=\frac{4-\left(\alpha_{d}+\alpha_{u}\right)\left(\alpha_{d}+\lambda \alpha_{u}\right)}{\left(1-\lambda \alpha_{u} \alpha_{d}\right)\left(1-\alpha_{u} \alpha_{d}\right)\left(\alpha_{d}-\lambda \alpha_{u}\right)}$, leading to $\Pi_{C}^{*}(0, \lambda)=\Pi_{M}^{*}(\lambda)$ (expression 10 in the text);

- $A(1, \lambda)=\frac{1}{2\left(t-\lambda \alpha_{u} \alpha_{d}\right)}, C(1)=\frac{1}{2}, D(1)=0$ and $\Delta(1, \lambda)=\frac{1}{\left(t-\lambda \alpha_{u} \alpha_{d}\right)\left(\alpha_{d}-\lambda \alpha_{u}\right)}$, leading to

$$
\Pi_{C}^{*}(1, \lambda)=\frac{t}{2}-\frac{\alpha_{d}^{2}}{16}-\frac{6 \alpha_{d} \alpha_{u} \lambda+\alpha_{u}^{2} \lambda^{2}}{16}
$$

which is equal to $\Pi_{C}^{*}(\lambda)$ (expression 11 in the text).

Calculating the derivatives of $\Pi_{C}^{*}(x, \lambda)$ in $x$ and $\lambda$ (for general $x$ and $\lambda$ ) is inextricable. We have used Mathematica to graph $\Pi_{C}^{*}(x, 0)$ and $\Pi_{C}^{*}(x, 1)$ as functions of $x$ and $\Pi_{C}^{*}\left(x_{0}, \lambda\right)$ as a function of $\lambda$ for various values of $x_{0}$ : see Figures 1 and 2 in Section 6.3.

\section{References}

[1] Ambrus, A. and R. Argenziano (2009) "Asymmetric Networks in Two-Sided Markets," American Economic Journal: Microeconomics, 1(1), 17-52

[2] Argenziano, R. (2007) "Differentiated Networks: Equilibrium and Efficiency," Rand Journal of Economics, 39(3), 747-769 
[3] Armstrong, M. (2006) "Competition in Two-Sided Markets," Rand Journal of Economics, 37 (3), 669-691

[4] Armstrong, M. and J. Wright (2007) "Two-sided Markets, Competitive Bottlenecks and Exclusive Contracts," Economic Theory, 32 (2), 353-380

[5] Caillaud, B. and B. Jullien (2003) "Chicken and Egg: Competition Among Intermediation Service Providers," Rand Journal of Economics, 34(2), 309-328

[6] Choi, J. P. (2010) "Tying in Two-Sided Markets With Multi-Homing", The Journal of Industrial Economics, 58(3), 607-626

[7] Church J. and N. Gandal (1992) "Network Effects, Software Provision and Standardization," Journal or Industrial Economics, 40(1), 85-103

[8] Economides, N. (1996) "Network externalities, complementarities, and invitations to enter," European Journal of Political Economy, 12 (2), 211-233

[9] Evans, D. and R. Schmalensee (2010) "Failure to Launch: Critical Mass in Platform Businesses," Review of Network Economics, 9(4), 1-26

[10] Farrell, J. and G. Saloner (1985) "Standardization, Compatibility, and Innovation," The RAND Journal of Economics, 16 (1), 70-83

[11] Farrell, J. and G. Saloner (1986) "Installed Base and Compatibility: Innovation, Product Preannouncements, and Predation," The American Economic Review, 76(5), 940955

[12] Gabszewicz, J. and X. Wauthy (2012) "Platform competition and vertical differentiation," CORE Discussion Papers, Université Catholique de Louvain, Center for Operations Research and Econometrics

[13] Griva, K. and N. Vettas (2011) "Price Competition in a Differentiated Products Duopoly Under Network Effects," Information Economics and Policy, 23, 85-97

[14] Hagiu, A. (2009) "Two-Sided Platforms: Product Variety and Pricing Structures," Journal of Economics \&3 Management Strategy, 18(4), 1011-1043. 
[15] Halaburda, H. and Y. Yehezkel (2013) "Platform Competition under Asymmetric Information," American Economic Journal: Microeconomics, 5(3), 22-68.

[16] Hart, O. and J. Tirole (1990) "Vertical Integration and Market Foreclosure," Brookings Papers on Economic Activity, 205-276

[17] Hurkens, S. and A. L. Lopez (2012) "Mobile Termination, Network Externalities, and Consumer Expectations," mimeo

[18] Katz, M. L. and C. Shapiro (1985) "Network externalities, competition, and compatibility," The American Economic Review, 75(3), 424-440

[19] Katz, M. L. and C. Shapiro (1986) "Technology Adoption in the Presence of Network Externalities," Journal of Political Economy, 94(4), 822-841

[20] McAfee, P. R. and M. Schwartz (1994) "Opportunism in Multilateral Contracting: Nondiscrimination, Exclusivity, and Uniformity," American Economic Review, 84, 210230

[21] Matutes, C. and X. Vives (1996) "Competition for Deposits, Fragility, and Insurance," Journal of Financial Intermediation, 5, 184-216

[22] Parker, G. and M. W. Van Alstyne (2005) "Two-Sided Network Effects: A Theory of Information Product Design," Management Science, 51, 1494-1504

[23] Rey, P. and T. Verge (2004) "Bilateral control with vertical contracts," Rand Journal of Economics, 35 (4), 728-746

[24] Rochet, J.-C. and J. Tirole (2003) "Platform Competition in Two-Sided Markets," Journal of the European Economic Association, 1 (4), 990-1029

[25] Rochet, J.-C. and J. Tirole (2006) "Two-Sided Markets: Where We Stand," Rand Journal of Economics, 37 (3), 645-66

[26] Weyl, E. G. (2010) "A Price Theory of Multi-sided Platforms," American Economic Review, 100(4), 1642-72 